Laurence lan C. Tan, MD'

Maria Rina T. Reyes-Quintos, MD, MClinAud ${ }^{1,2}$

Maria Leah C. Tantoco, MD, MClinAud ${ }^{2}$

Charlotte M. Chiong, MD ${ }^{1,2}$

'Department of Otorhinolaryngology

Philippine General Hospital

University of the Philippines Manila

${ }^{2}$ Philippine National Ear Institute

National Institutes of Health

University of the Philippines Manila
Correspondence: Laurence lan C. Tan, MD

Department of Otorhinolaryngology

Ward 10, Philippine General Hospita

Taft Avenue, Ermita, Manila 1000

Philippines

Phone: (632) 5264360

Fax: (632) 5255444

Reprints will not be available from the author.

Funding for this study was supported by Hearing International and the Philippine National Ear Institute. Hearing International provided the training for ASSR as well as financial support for purchase of the equipment. The Philippine National Ear Institute provided the venue for testing of the children and also partly funded the research by way of salaries of the data collectors. The authors signed a disclosure that they have no proprietary or financial interest with any other organizations that may have a direct interest in the subject matter of this manuscript, or in any product used or cited in this study.

Presented at the Analytical Research Contest (1st Place) Philippine Society of Otolaryngology Head and Neck Surgery, Glaxo-Smith-Kline Bldg., Auditorium, Chino Roces Avenue, Makati City. October 23, 2008

\section{Comparative Study of the Auditory Steady-State Response (ASSR) and Click Auditory Brainstem- Evoked Response (click ABR) Thresholds among Filipino Infants and Young Children}

\begin{abstract}
Objective: To compare the results of auditory steady-state response (ASSR) and click auditory brainstem response (click ABR) among infants and young children tested at the Ear Unit of a Tertiary General Hospital.
\end{abstract}

\section{Methods:}

Design: Cross-sectional Study

Setting: Tertiary General Hospital

Population: Within-subject comparisons of click auditory brainstem response (click ABR) thresholds and auditory steady-state response (ASSR) thresholds among 55 infants and young children, 2 months to 35 months of age referred to the Ear Unit for electrophysiologic hearing assessment.

Results: Click ABR showed strong positive correlation to all frequencies and averages of ASSR. Highest correlation was noted with the average of $1-4 \mathrm{kHz}$ ASSR results with Pearson $r=$ 0.89 (Spearman $r=0.80$ ), the average of $2-4 \mathrm{kHz}$ had strong positive correlation $r=0.88(0.79)$. Correlation was consistently strong through all ASSR frequencies $(0.5 \mathrm{kHz}$ at $r=0.86(0.74), 1 \mathrm{kHz}$ at $r=0.88(0.78), 2 \mathrm{kHz}$ at $r=0.87(0.79), 4 \mathrm{kHz}$ at $r=0.85(0.76))$. Average differences of click $A B R$ and ASSR thresholds were $8.2 \pm 12.9 \mathrm{~dB}$ at $0.5 \mathrm{kHz}, 8.6 \pm 12.6 \mathrm{~dB}$ at $1 \mathrm{kHz}, 5.3 \pm 11.8 \mathrm{~dB}$ at $2 \mathrm{kHz}$ and $7.8 \pm 13.4 \mathrm{~dB}$ at $4 \mathrm{kHz}$. Among patients with no demonstrable waveforms by click $A B R$ with maximal click stimulus, a large percentage presented with ASSR thresholds. Of these, $80.5 \%$ (33 of 41) had measurable results at $0.5 \mathrm{kHz}$ with an average of $107.3 \pm 11.1 \mathrm{~dB}, 85.4 \%$ (35 of 41 ) at $1 \mathrm{kHz}$ with an average of $110.5 \pm 11.8 \mathrm{~dB}, 73.2 \%$ ( 30 of 41 ) at $2 \mathrm{kHz}$ with an average of $111.2 \pm 11.1 \mathrm{~dB}$ and $63.4 \%$ ( 26 of 41 ) at $4 \mathrm{kHz}$ with average of $112.2 \pm 8.21 \mathrm{~dB}$. Auditory steady-state response results were comparable to auditory brainstem response results in normal to severe hearing loss and provided additional information necessary for complete audiologic assessment especially among patients with severe to profound hearing loss wherein click ABR showed no responses. Up to $85.4 \%$ of patients that would have been noted to have no waveforms by click ABR still demonstrated measurable thresholds by ASSR.

Conclusion: Our study suggests that ASSR may be the best available tool for assessing children with severe to profound hearing loss, and is a comparably effective tool in overall hearing assessment for patients requiring electrophysiological testing. The advantages of ASSR over click ABR include: 1) detection of frequency-specific thresholds and; 2) the detection of hearing loss thresholds beyond the limits of click ABR.

Key words: Auditory Steady-State Response, ASSR, Auditory Brainstem-Evoked Response, ABR, Hearing Thresholds, Electrophysiologic Testing

Philipp J Otolaryngol Head Neck Surg 2009; 24 (1): 9-12

(C) Philippine Society of Otolaryngology - Head and Neck Surgery, Inc. 


\section{ORIGINAL ARTICLES}

Hearing screening has been employed in the Philippines for more than a decade with recent efforts to promote universal newborn hearing screening. Despite detrimental effects of childhood hearing loss documented among Filipino children, ${ }^{1}$ we still lack widespread use of newborn and infant hearing screening programs. Advocacy and active promotion have resulted in increasing popularity of the use of otoacoustic emissions (OAE) and auditory brainstem-evoked response (ABR). However, auditory steady-state response (ASSR) has only recently become available in the Philippines despite its well established use. For instance, it has been incorporated in hearing screening programs in the United Kingdom since 2007.2

Auditory Steady-State Response (ASSR) and Auditory Brainstem Response (ABR) are both electrophysiologic tests with very similar basic principles. The EEG waveforms generated from the auditory stimulus are assumed to correspond to certain portions of the auditory brainstem pathway. The presence or absence of these waveforms in response to a stimulus determines the estimated threshold of the patient for ABR.

Click $A B R$ is still the most commonly used electrophysiologic test to evaluate the auditory pathway's integrity among infants and young children. Click stimuli used for $A B R$ are broad frequency over the spectrum $1 \mathrm{k}-4 \mathrm{kHz}$. ${ }^{3}$ Tone-burst ABR has been shown to have good correlation for low frequency range of $250 \mathrm{~Hz} .{ }^{4}$ Tone-burst $A B R$ and ASSR have frequency specific stimuli and have been shown to have accurate correlation. ${ }^{5}$ Tone-burst ABR's need for new instrumentation and technical expertise has limited it's application locally.

Moreover, $A B R$ requires experience in waveform analysis and can be prone to reader error. ASSR employs an objective, sophisticated, statistics-based mathematical detection algorithm to detect and define hearing thresholds. This objectivity provides an added advantage over ABR.

Several studies have confirmed the correlation of ABR to ASSR results, ${ }^{5,6,7,8,9}$ as well as correlation to pure-tone thresholds. ${ }^{10,11,12}$ Hearing loss beyond $95 \mathrm{~dB}$ are beyond the limits for $A B R$ stimulus presentation. Compared to the limitation of click ABR, ASSR signal intensity can be as high as $120 \mathrm{~dB}$.

Despite the acceptance of ASSR as a diagnostic test in the evaluation of hearing loss, there has been no local investigation on ASSR in Filipino hearing-impaired children. The results of this study can be used to estimate thresholds in cases where ABR flat waves have been recorded. These will provide information that may be important for counseling parents and clinicians regarding the rationale for hearing aid fitting and its settings in patients who would have been deprived of knowing the extent of residual hearing in areas where as yet no ASSR services are available.

The objectives of this study are 1) to compare the results of ASSR and click ABR among Filipino infants and young children tested the Ear Unit in the Philippine General Hospital; 2) to correlate the click ABR and ASSR and; 3 ) to describe the ASSR results among patients with nonreactive waveforms on maximal click $A B R$ stimulus.

\section{Subjects}

\section{METHODS}

All patients who failed a hearing screening and who were referred to the Ear Unit of the Philippine General Hospital for electrophysiologic testing were considered and informed consent was obtained. Excluded were patients who could not undergo electrophysiological testing either due to external ear abnormalities, inability to be fit with electrodes/ear inserts or undergo sedation. Fifty five patients were included, aged 2 months to 35 months. The majority of patients referred could not undergo behavioral testing.

Hearing assessment was conducted in a soundproof room. Electrophysiological audiologic assessment was performed in the same session and administered by the same tester. Patients were tested under sedation using chloral hydrate or in natural sleep state when consent for sedation was not given.

\section{ABR stimulation and recording}

Click ABRs were recorded using the Bio-logic MASTER system (Biologic Systems Corporation, Mundelein, IL). Click ABRs were measured with electrodes affixed to the vertex and to the mastoid processes. Either the forehead or the contralateral mastoid process served as ground. Electrode impedances never exceeded $3000 \mathrm{~m} \Omega$.

Responses were measured to $100 \mathrm{~ms}$ rarefaction clicks presented monaurally. ABRs were obtained initially at $30 \mathrm{~dB}$ or $70 \mathrm{~dB}$ depending on the clinical presentation of the patient. One thousand twenty four stimulus presentations were included in each average response which was replicated at least once. Waveforms recorded with artifacts of movement were re-sampled. A $10 \mathrm{~dB}$ increment or decrement was used to determine the threshold. The threshold was determined at the lowest level at which an ABR Wave $V$ was present as determined by visual inspection of the waveforms displayed on the computer screen.

\section{ASSR stimulation and recording}

ASSR testing immediately followed the click ABR for patients who were still asleep or sedated. The same surface electrodes used in ABR were also used for ASSR, which was measured using a predefined program of the same Bio-logic MASTER apparatus. Patients were tested at $10 \mathrm{~dB}$ below previously determined $A B R$ thresholds when available. Increments/decrements of $10 \mathrm{~dB}$ were used depending on the required number of sweeps per frequency and threshold. Patients tested for thresholds of $80 \mathrm{~dB}$ and above were tested monaurally and one frequency at a time. The MASTER system takes into account the variance of the noise along with the variance of the response and determines significance using an F-test.

\section{RESULTS}

Fifty five patients, aged 2 months to 35 months with mean age at $18 \pm 9$ months were included in this study (Table 1). One patient was tested unilaterally due to aural atresia which prevented placement of ear inserts in the affected ear. Five patients woke up during ABR testing, completing only one of the ears tested. One patient did not complete ASSR testing for one ear. A total of 103 ears were tested for both ABR 
and ASSR. ABR test results were compared to ASSR results at $500 \mathrm{~Hz}$, $1,000 \mathrm{~Hz}, 2,000 \mathrm{~Hz}$ and $4,000 \mathrm{~Hz}$ when available.

Statistical analysis was done with Pearson and Spearman correlation using GraphPad Prism version 5.00 for Windows, (GraphPad Software, San Diego California USA,www.graphpad.com). Table 2 lists the correlation data and values. ABR showed strong positive correlation to all frequencies and averages of ASSR. Highest correlation was noted with the average of 1-4 kHz ASSR results (Figure 1) with Pearson $r=0.89$ (Spearman $r=0.80$ ), the average of $2-4 \mathrm{kHz}$ (Figure 2) had strong positive correlation $r=0.88(0.79)$. These results, however, are less than those determined by previous studies (Pearson $r=0.92) . .^{13}$ Correlation was consistently strong through all ASSR frequencies (Table 2, Figure 3). All correlations were significant at a 0.01 level of significance (2 - tailed).

Differences between the click ABR and ASSR thresholds were also noted (Table 3). The average differences of click ABR and ASSR thresholds were $8.2 \pm 12.9 \mathrm{~dB}$ for $0.5 \mathrm{kHz}, 8.6 \pm 12.6 \mathrm{~dB}$ at $1 \mathrm{kHz}, 5.3 \pm 11.8 \mathrm{~dB}$ at $2 \mathrm{kHz}$ and $7.8 \pm 13.42 \mathrm{~dB}$ at $4 \mathrm{kHz}$.

Forty-one patients were non-responsive (NR) or exhibited no recognizable waveforms with maximal stimulus by click $A B R$. Two test ears were non-responsive (NR) by both click ABR and ASSR. Of 41 test ears non-responsive (NR) by click ABR, 39 had results with ASSR for at least one frequency.

Among patients with no demonstrable waveforms by click $A B R$, a large percentage presented with ASSR thresholds. Of these, 80.5\% (33 of 41 ) had measurable results at $0.5 \mathrm{kHz}$ with a mean of $107.3 \pm 11.1 \mathrm{~dB}$, $85.4 \%$ (35 of 41 ) at $1 \mathrm{kHz}$ with an average of $110.5 \pm 11.8 \mathrm{~dB}, 73.2 \%$ (30 of 41 ) at $2 \mathrm{kHz}$ with a mean of $111.2 \pm 11.1 \mathrm{~dB}$ and $63.4 \%$ ( 26 of 41 ) at 4 $\mathrm{kHz}$ with mean of $112.2 \pm 8.2 \mathrm{~dB}$ (Figure 4 ).

\section{DISCUSSION}

This study confirmed that ASSR is comparable to click ABR as a measure of hearing thresholds for Filipino children. The highest correlation was found to be with the average of $1-4 \mathrm{kHz}$ followed by the average of $2-4 \mathrm{kHz}$ due to the nature of the click stimulus being within the high frequency range. These results were very similar to those demonstrated in 48 infants and young children by Swanepoel and Ebrahim. ${ }^{8}$ The click stimulus, as described earlier, does recruit the cochlear range of $1-4 \mathrm{k}$. It could be recommended that click $A B R$ be compared to the averages of high frequency ASSR rather than to a single frequency.

The means of the differences (Table 4) between click ABR and ASSR test frequencies were minimal (less than $10 \mathrm{~dB}$ ), which is the increment used in clinical practice. With the hearing thresholds $0-40 \mathrm{~dB}$ and 61 $80 \mathrm{~dB}, \mathrm{ASSR}$ averages were generally higher by approximately $10 \mathrm{~dB}$ and $15 \mathrm{~dB}$. These differences varied in the higher sound intensities. ASSR much more closely approached thresholds obtained by click $A B R$ in the severe to profound hearing levels, though the limited number of patients with recordable thresholds by click ABR may have influenced results greatly. The over-estimation of thresholds may have been influenced by machine calibration or affected by the statistical elimination of EEG noise which is especially prominent among lower intensity thresholds. ${ }^{13}$ These results of over-estimation of thresholds

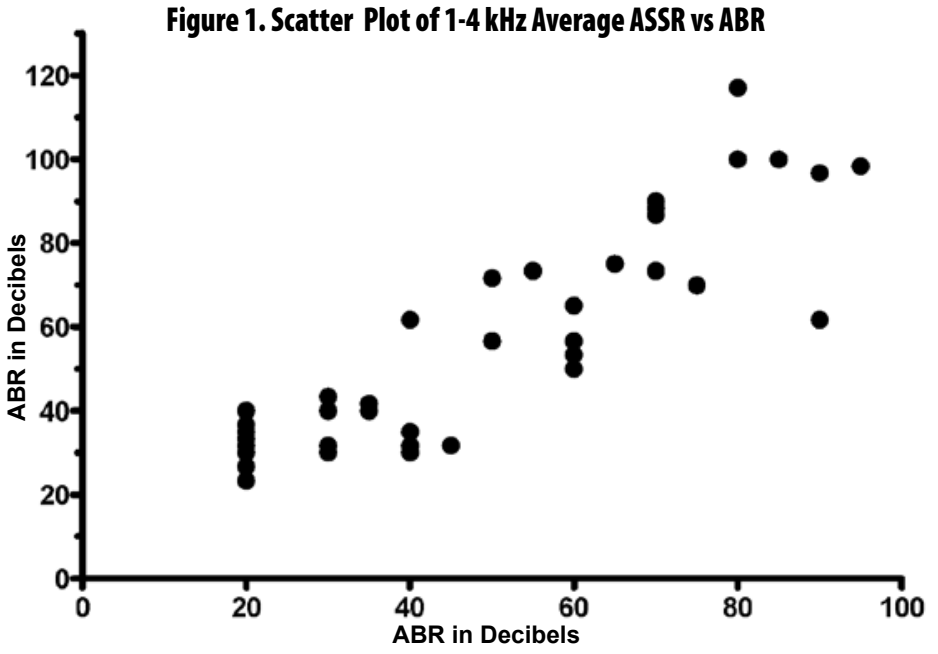

Figure 1. Shows the distribution of the average threshold of 1-4 kHz ASSR against the ABR.

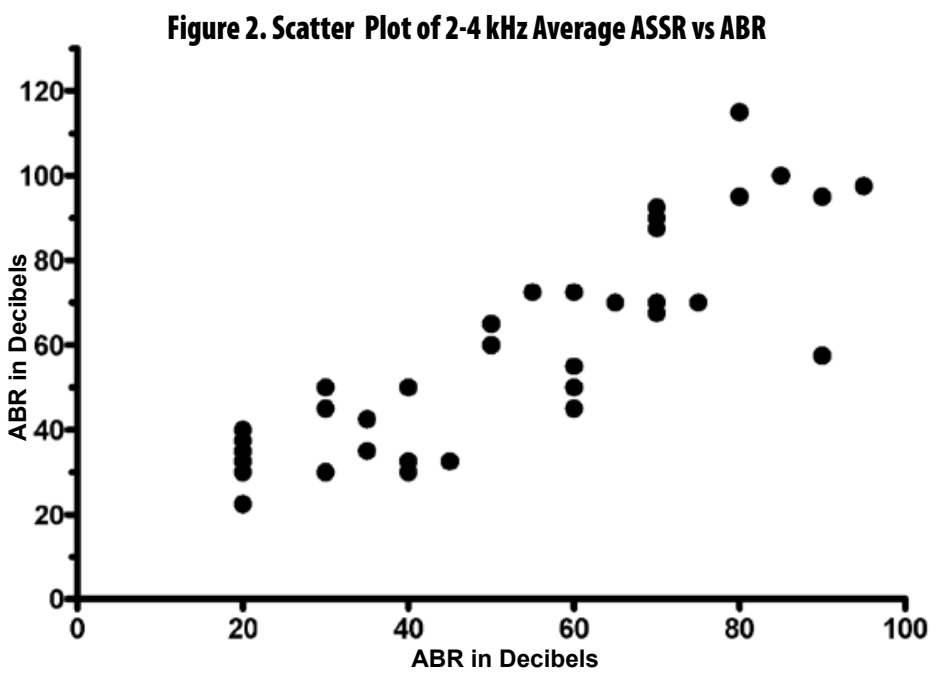

Figure 2. Shows the distribution of the average threshold of $2-4 \mathrm{kHz}$ ASSR against the ABR.

Figure 3. Scatter Plot of ASSR Frequencies vs ABR

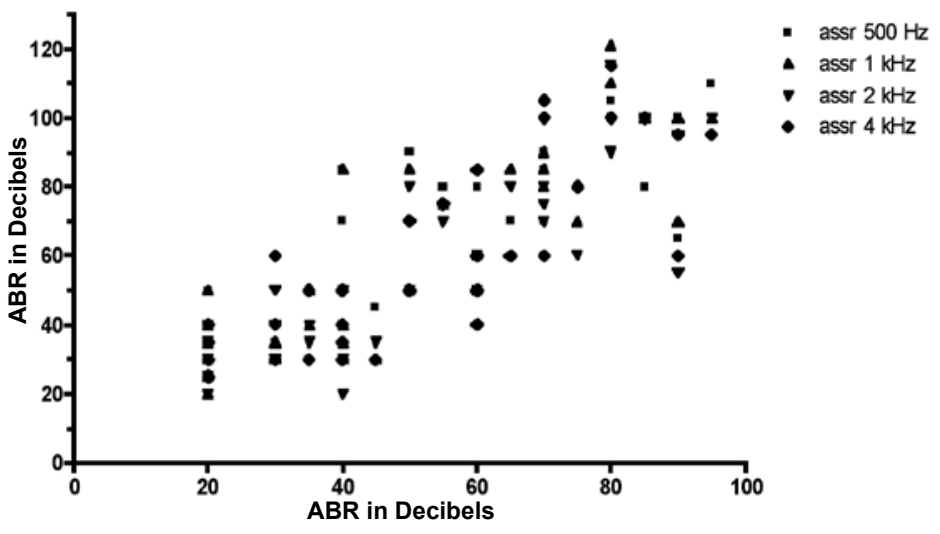

Figure 3. Shows the distribution of the ASSR against the ABR. 
Table 1. Classification of Patients' Hearing

\begin{tabular}{|ccc|}
$\begin{array}{c}\text { Hearing Status } \\
\text { (ABR thresholds) }\end{array}$ & Total No. of Ears & $\begin{array}{c}\text { Average Age } \\
\text { Months }\end{array}$ \\
\hline $0-40 \mathrm{~dB}$ & 41 & $15 \pm 9$ \\
$41-60 \mathrm{~dB}$ & 8 & $11 \pm 7$ \\
$61-80 \mathrm{~dB}$ & 9 & $18 \pm 10$ \\
81 and above dB & 4 & $22 \pm 8$ \\
No Response & 41 & $22 \pm 8$ \\
All thresholds & 103 & $18 \pm 9$ \\
\hline
\end{tabular}

Table 2. Correlation of click ABR to ASSR thresholds

\begin{tabular}{lcccccc} 
& $\mathbf{0 . 5} \mathbf{~ k H z}$ & $\mathbf{1 ~ k H z}$ & $\mathbf{2 ~} \mathbf{~ H z}$ & $\mathbf{4 ~} \mathbf{~ H z}$ & $\mathbf{1 - 4} \mathbf{~ k H z}$ & $\mathbf{2 - 4} \mathbf{~ k H z}$ \\
Pearson Correlation & .86 & .88 & .87 & .85 & .89 & .88 \\
Spearman Correlation & .74 & .78 & .79 & .76 & .80 & .79 \\
Sig. (2-tailed) & .00 & .00 & .00 & .00 & .00 & .00 \\
$\mathrm{~N}$ & 62 & 62 & 62 & 62 & 62 & 62 \\
\hline
\end{tabular}

Table 3. Mean Differences between ASSR and click ABR

\begin{tabular}{l|r|l|l|l|l|l|l|}
\multirow{2}{*}{$\begin{array}{l}\text { Hearing } \\
\text { thresholds }\end{array}$} & \multicolumn{7}{|c|}{ Difference in decibels (dB) } \\
\cline { 2 - 8 } $0-40 \mathrm{~dB}$ & 41 & $\mathbf{0 . 5} \mathbf{~ k H z}$ & $\mathbf{1 ~ k H z}$ & $\mathbf{2 ~ k H z}$ & $\mathbf{4 ~ k H z}$ & $\mathbf{1 - 4} \mathbf{~ k H z}$ & $\mathbf{2 - 4} \mathbf{~ k H z}$ \\
$41-60 \mathrm{~dB}$ & 8 & $8.1 \pm 11.6$ & $8.2 \pm 10.9$ & $6.2 \pm 9.5$ & $8.3 \pm 9.3$ & $7.6 \pm 8.8$ & $7.3 \pm 8.9$ \\
$61-80 \mathrm{~dB}$ & 9 & $10 . \pm 11.2$ & $17.3 \pm 13$ & $8.9 \pm 13.2$ & $15 \pm 20$ & $13.7 \pm 12.4$ & $11.9 \pm 13.4$ \\
$81 \mathrm{~dB}$ and above & 4 & $-1.2 \pm 18.0$ & $2.5 \pm 15.6$ & $-2.5 \pm 22.2$ & $-2.5 \pm 19.4$ & $-0.8 \pm 19.0$ & $-2.5 \pm 20.7$ \\
All Thresholds & 62 & $8.2 \pm 12.9$ & $8.6 \pm 12.6$ & $5.3 \pm 11.8$ & $7.8 \pm 13.4$ & $7.2 \pm 11.0$ & $6.6 \pm 11.4$ \\
\hline
\end{tabular}

Table 4. Comparison of click ABR to ASSR

\begin{tabular}{|c|c|c|}
\hline & Click ABR & ASSR \\
\hline $\begin{array}{l}\text { Detection of Retrocochlear } \\
\text { Pathology }\end{array}$ & \multirow{3}{*}{$\begin{array}{l}\text { Sensitivity of }>90 \%{ }^{14} \\
\text { Identified with wave I } \\
\text { Yes }^{15}\end{array}$} & \multirow{3}{*}{$\begin{array}{l}\text { No studies available } \\
\text { Cannot differentiate sensory from } \\
\text { neural } \\
\text { No }\end{array}$} \\
\hline Neural/Auditory Neuropathy & & \\
\hline Intraoperative Monitoring & & \\
\hline Estimation of Hearing Thresholds & & \\
\hline Normal hearing & Accurate & $\begin{array}{l}\text { Tendency for over-estimation if } \\
\text { patient not sedated }\end{array}$ \\
\hline Severe to profound hearing loss & $\begin{array}{l}\text { Accurate only to moderate HL } \\
\text { Limited to 95dB15 }\end{array}$ & $\begin{array}{l}\text { Accurate from moderate to } \\
\text { profound HL }\end{array}$ \\
\hline Ski slope hearing & $\begin{array}{l}\text { Results not representative of } \\
\text { hearing loss }\end{array}$ & Frequency-specific thresholds \\
\hline Frequency range & $1-4 \mathrm{kHz}$ & $0.25-4 \mathrm{kHz}$ \\
\hline Simultaneous testing & No & Up to 8 frequencies at a time ${ }^{13}$ \\
\hline
\end{tabular}

are consistent with results from several studies. ${ }^{11,12}$ The approach of the average ASSR threshold to the click ABR may also be due to the effect of saturation of ASSR thresholds noted at higher frequencies when using sweeps of intensities as noted by Picton. ${ }^{13}$

For patients without demonstrable ABR waveforms at maximal click intensity, a large percentage showed residual hearing at $500 \mathrm{~Hz}$ at a mean close to the limit of the ABR. From $1 \mathrm{k}$ to $4 \mathrm{kHz}$, there were a decreasing number of patients with responses to ASSR. These results best demonstrate the limitation of testing with $A B R$ for patients with profound hearing loss in the range of the click stimulus. Notably, up to $85.4 \%$ of patients that would have been noted to have no waveforms by click ABR still demonstrated measurable thresholds by ASSR. Figure 4 shows the results of patients that still had ASSR thresholds but had no waveforms by click ABR. The majority of thresholds were distributed at $110-120 \mathrm{~dB}$ but the variation of ASSR thresholds varied widely especially among the lower frequencies. It may therefore be necessary that patients noted to have no waveforms by ABR be crosschecked by ASSR. The recommendation is that ASSR testing be done in patients who fail to demonstrate waveform responses by click ABR.

Our study suggests that ASSR may be the best available tool for assessing children with severe to profound hearing loss and is a comparably effective tool in overall hearing assessment for patients requiring electrophysiological testing. The advantages of ASSR over click ABR (Table 4) include: 1) detection of frequency-specific thresholds and;2) the detection of hearing loss thresholds beyond the limits of click ABR. However, it is our view that ASSR not be taken as a replacement for the click $A B R$ but as a complement to the audiologic armamentarium.

\section{REFERENCES}

1. Chiong C, Ostrea E Jr, Reyes A, Llanes EG, Uy ME, Chan A. Correlation of hearing screening with developmental outcomes in infants over a 2-year period. Acta Otolaryngol. 2007 Apr;127(4):384-

2. Stevens J, Sutton G, Wood S, Mason S. Guidelines for the early audiological assessment and management of babies referred from the newborn hearing screening programme. [Database on the Internet] Newborn Hearing Sscreening Program c2002-2005 - [update 2007 March; cited 2008 September 3]. Available from:http://hearing.screening.nhs.uk.

3. Ponton C. W.; Moore J. K.; Eggermont J. J.; Auditory brain stem response generation by parallel pathways : Differential maturation of axonal conduction time and synaptic transmission. Ear and hearing 1996:(17):402-410.

4. Gorga M. P.; Johnson T. A.; Kaminski J. R.; Beauchaine K. L.; Garner C. A.; Neely S. T.; Using a combination of click- and tone burst-evoked auditory brainstem response measurements to estimate pure-tone thresholds. Ear and hearing 2006 Feb;27(1):60-74.

5. Johnson T. A.; Brown C. J.; Threshold prediction using the auditory steady-state response and the tone burst auditory brainstem response: a within subject comparison. Ear and hearing 2005 Dec; 26(6):559-76.

6. Lin Y-H, Ho HC, Wu HP, Comparison of auditory steady-state responses and auditory brainstem responses in audiometric assessment of adults with sensorineural hearing loss, Auris Nasus Larynx (2008), 2008 Jul 11. [Epub ahead of print]

7. Firszt J, Gaggl W, Runge-Samuelson C, Burg L, Wackym P. Auditory Sensitivity in Children Using the Auditory Steady-State Response Arch Otolaryngol Head Neck Surg. 2004;130:536-540.

8. Swanepoel D, Ebrahim S Auditory steady-state response and auditory brainstem response thresholds in children Eur Arch Otorhinolaryngol 2008 June 17 [Epub ahead of print].

9. Rance G, Tomlin D, and Rickards F. Comparison of Auditory Steady-State Responses and ToneBurst Auditory Brainstem Responses in Normal Babies Ear \& Hearing 2006; 27: 751-762.

10. Swanepoel D, Hugo R,Roode R. Auditory Steady-State Responses for Children With Severe to Profound Hearing Loss Arch Otolaryngol Head Neck Surg. 2004;130:531-535.

11. Duarte JL, Alvarenga KF, Garcia TM, Costa Filho OA, Lins OG. Auditory steady-state response in the auditory evaluation: clinical application (original title: A resposta auditiva de estado estável na avaliação auditiva: aplicação clínica). Pró-Fono Revista de Atualização Científica. 2008 abrjun;20(2):105-10.

12. Herdman AT, Stapells DR. Thresholds determined using the monotic and dichotic multiple auditory steady-state response technique in normal-hearing subjects. Scand Audiol. 2001;30(1):41-9.

13. Picton TW, Roon P, and John MS. Human Auditory Steady-State Responses During Sweeps of Intensity. Ear \& Hearing 2007;28:542-557.

14. Zappia JJ, O"Connor CA, Wiet RJ, Dinces EA. Rethinking the use of auditory brainstem response in acoustic neuroma screening. Laryngoscope. Oct 1997;107(10):1388-92

15. Katz J. Handbook of Clinical Audiology, Fourth Edition. Baltimore, Md:. Lippincott Williams \& Wilkins;1994. 